\title{
Anesthetic management of a patient with myotonia congenita
}

\author{
Syed Shakir Hasan
}

West Cumbria Hospital, Homewood Road, Hensingham, Whitehaven CA28 8JG, United Kingdom

Correspondence: Dr. Syed Shakir Hasan; E-mail: shakirhasanpk@yahoo.com; Phone: 00447432605854; Mobile: 447432605854

\section{Abstract}

Myotonia congenita is also known as Becker's disease. It is a genetic disorder in which skeletal muscles are unable to relax quickly after voluntary movements. Symptoms may vary from person to person and may include muscle stiffness and weakness initiated by movement after rest.

Myotonia congenita was first described by Thomsen in 1876 as a disorder of skeletal muscles. Patients suffer from muscle contractility and fatigue which occurs as a consequence of the mutation of the chloride channel gene on the 17th chromosome. These patients have a high risk of developing malignant hyperthermia during anesthesia, and call for full preparation to prevent and deal with this complication.

Key words: Becker's disease; Myotonia congenita; Anesthesia; Malignant hyperthermia

Citation: Hasan SS. Anesthetic management of a patient with myotonia congenita. Anaesth. pain intensive care 2021;25(6):816-818 ; DOI: 10.35975/apic.v25i6.1712

Received: August 25, 2021, Reviewed: September 19, 2021, Accepted: October 03, 2021

\section{Introduction}

Myotonia congenita (MC), also known as Becker's disease, is a rare genetic disorder of skeletal muscles, which can cause hyper excitability on stimulation. The disease may present with muscular hypertrophy, muscle contraction and muscle weakness, which can lead to the symptoms such as dysphasia, aspiration and cardiomyopathy. The problems that can occur with this condition during anesthesia include masseter spasm at induction leading to difficulty in intubation, aspiration, persistent myotonic contraction and inability of the muscles to relax. The condition can lead to severe acidosis and hyperkalemia. The most serious threat is the possibility of the onset of malignant hyperthermia.

Although this is a rare condition, some of the anesthetists may encounter it during their practice and face the challenge of dealing with it. Its association with anesthesia can be catastrophic; therefore, it is important to have a detailed preoperative assessment of these patients, and to have an elaborate management plan. The choice of anesthesia is important; it is recommended to choose a regional anesthesia whenever possible. If regional anesthesia is not possible, then total intravenous anesthesia (TIVA) is an alternative option. In our case we had the option to choose spinal anesthesia. Inhalational anesthesia can lead to shivering, and therefore can cause myotonia and it is also one of two main offenders for developing malignant hyperthermia (MH) along with suxamethonium. Non-depolarizing muscle relaxants are not contraindicated in this condition, but should be used cautiously. Sugammadex for reversal is a better choice than neostigmine as the later may induce myotonia. Therefore, TIVA with propofol and remifentanil is a good alternative to regional anesthesia. Care should be taken to avoid using offenders of $\mathrm{MH}$ and to prevent the triggering factors for developing myotonia. We present our experience of anesthetic management in a patient with MC.

\section{Case report}

A 46 y old female was scheduled for total abdominal hysterectomy. Her past medical history comprised of MC, diagnosed on EMG at the age of 12 years, involving her lower limbs. She had had some corrective surgeries done on her feet as a child, to deal with the deformity associated with muscular hypertrophy. She was under the care of the neurologist for her condition and didn't seem to have any muscle weakness or muscle contraction before the surgery. She also had mild asthma. 
She was seen in the pre-operative anesthesia clinic and a red flag was raised regarding her $\mathrm{MC}$ and its associated risks with anesthesia. Information about her ailment and the proposed date of surgery was relayed to the concerned anesthetist. Patient was initially planned by the surgeon for laparoscopic abdominal hysterectomy but he changed the procedure to total abdominal hysterectomy after coming to know the risks associated with laparoscopy in this patient. After the discussion between the anesthetist and the surgeon, spinal anesthesia was planned as it was considered a much safer option compared to general anesthesia. On the day of surgery, the anesthetist who had been involved in all the discussions and planning with the surgeon pre-operatively, happened to have a day off and the list was assigned to another anesthetist.

The anesthesia machine vaporizer was removed for $2 \mathrm{~h}$ and the machine was flushed with oxygen for $30 \mathrm{~min}$. Dantrolene was made available in the operating room and its dose was calculated as per body weight. An assistant was assigned to prepare the drug, should the need arose.

The standard monitors were attached to the patient. Temperature of the patient was recorded before induction to have a baseline reading. Patient's vital signs were within normal range.

Spinal anesthesia was planned for this patient as plan A and TIVA as plan B.

Inj. bupivacaine $0.5 \%$ heavy $3.2 \mathrm{ml}+$ inj. fentanyl $25 \mu \mathrm{g}$ were given into the intrathecal space. Diamorphine and morphine were not available in our operating rooms on that day. Inj. midazolam $3 \mathrm{mg}$ was administered intravenously for sedation. The height of the spinal block was recorded to be T6 bilaterally. The surgery lasted for about two hours, and the patient remained stable hemodynamically. Everything went very well until 15 min before the end of the surgery, when the patient started feeling pain. In. fentanyl $100 \mu \mathrm{g}$ was administered IV to relieve the pain without much success, and then it was followed by propofol $1 \%$ infusion in sedative dose. Patient got settled with this for the rest of the surgery, breathing spontaneously with oxygen supplement at 6 $\mathrm{L} / \mathrm{min}$ via face mask and remained rousable. We didn't notice any increase in muscle tone at any point of time during surgery.

The patient was kept warm with the help of the warming blanket and warm fluids to prevent shivering. Her body temperature and other vital parameters, such as blood pressure and heart rate, remained normal. She required inj. morphine IV in the recovery room and was transferred to the ward on morphine patient controlled analgesia (PCA).

\section{Discussion}

$\mathrm{MC}$ is a condition that has two types, Thomsen disease and Becker disease. Thomsen disease is a less severe variant than the Becker disease. The later involves lower limbs mainly. There is a high risk of developing masseter spasm and/or malignant hyperthermia on induction of general anesthesia. Certain other factors, such as shivering and cold environment, can trigger myotonia and can lead to complications. The choice of anesthesia in this condition is preferred to be a regional anesthesia; however, if general anesthesia is unavoidable then TIVA is a technique of choice.

Our patient had Becker type MC. Her symptoms were well controlled on medication. She was well planned ahead of her surgery and a spinal anesthesia was chosen for definitive reasons. TIVA was kept as a plan B if the plan A failed.

Other triggering factors for the development of myotonia were taken well care of by the use of a warm blanket and warm fluids. Frequent temperature monitoring was also done to identify a rise or drop in the body temperature early. A rising temperature might indicate a likely development of $\mathrm{MH}$ and a drop could lead to shivering which could precipitate myotonia.

Although the plan was to use spinal anesthesia, yet it was ensured that dantrolene was available in the operating room along with an assigned person to prepare and administer it should the need arose. The patient had a good sedation and the surgery went on smoothly. However, there was a brief period at the end of surgery when the patient felt some discomfort and pain, which was managed successfully with the administration of fentanyl and propofol infusion in sedative doses.

Following drugs can be administered safely;

- Induction agents such as etomidate, thiopentone and propofol, but need to be used in reduced doses.

- Opioids can be used cautiously, and in reduced doses due to their respiratory depressant effect.

- NSAIDS \& acetaminophen.

- Shorter acting non-depolarizing muscle relaxant in reduced doses.

\section{- Sugammadex.}

Following drugs are potentially unsafe;

- Inhalational agents

- Suxamethonium

- Neostigmine

\section{Conclusion}

Myotonia congenita poses a real safety threat during anesthesia, especially during general anesthesia of 
developing multiple complications including malignant hyperthermia. It might be catastrophic if someone is encountered with an undiagnosed case or during an emergency surgery. All elective cases need a comprehensive preoperative evaluation of the patient and a meticulous planning in order to achieve a good outcome. The preferable choice of anesthesia is regional anesthesia. TIVA might have to be used with utter care and vigilance.

\section{Conflict of interest}

None declared by the authors.

\section{Authors' contribution}

SSH managed the case and is the sole author of this case report.

\section{References}

1. Cokay Abut $Y$, Şimşek S, Köse S, Şenay Kırgezen, Bolat K, Erden V. Anesthesia experience in a patient with myotonia congenita. Bagcilar Med Bull. 2019;4(1):53-55. DOI: 10.4274/BMB.galenos.2019.28989

2. Farbu E, Søfteland E, Bindoff LA. Anaesthetic complications associated with myotonia congenita: case study and comparison with other myotonic disorders. Acta Anaesthesiol Scand. 2003 May;47(5):630-4. [PubMed] DOI: 10.1034/j.13996576.2003.00116.x

3. Lee PG, Sachin Gupta S. Management of a patient with myotonia congenita undergoing patella ORIF. Proceedings UCLA Health. 2019;23. [FreeFullText]

4. Mossad H, Ortiz EM. An overview of myotonia congenita, rare disease, genetic disorder. 2020. 